\title{
The Effect of Different Agricultural Wastes on Aroma Composition of Shiitake (Lentinula edodes (Berk.) Pegler) Mushroom
}

\author{
Gökhan Baktemur ${ }^{1, a, *}$, Zeynep Dilan Çelik ${ }^{2, b}$, Ecem Kara ${ }^{1, c}$, Hatıra Taşkın ${ }^{1, d}$ \\ ${ }^{I}$ Department of Horticulture, Faculty of Agriculture, Cukurova University, 01330 Adana, Turkey \\ ${ }^{2}$ Department of Food Engineering, Faculty of Agriculture, Cukurova University, O1330 Adana, Turkey
}

${ }^{*}$ Corresponding author

A R T I C L E I N F O A B S T R A C T

Research Article

Shiitake mushroom (Lentinula edodes (Berk.) Pegler) popular as both edible and medicinal, is one of the most cultivated and consumed mushroom species in the world. Cultivation of this mushroom on different agricultural wastes has been experimented in many studies until today. Nevertheless, agricultural waste trials are going on as agricultural production patterns of the countries are

Received : 04/03/2020

Accepted : 10/04/2020 different. In this study, volatile aroma composition of shiitake mushroom produced on different agricultural wastes (wheat stalk, wheat bran, peanut shell, corn cob and vine pruning waste) was compared. Oak sawdust was selected as control because of its common use in literature and substrate materials were mixed at different ratios. In addition, some substrate mixtures were prepared with poplar sawdust. Volatile compounds of the shiitake mushroom were determined by using headspace-solid phase microextraction (HS-SPME) technique combined with Gas

Keywords:

Shiitake

Lentinula edodes

Volatile aroma composition

Agricultural wastes

Sulphur containing components
Chromatography-Mass Spectrometer (GC-MS). At the end of study, 41 volatile compounds were detected and dimethyl trisulfide, benzaldehyde, dimethyl disulfide, 1-octen-3-ol and 3-octanone were found to be dominant volatile components. Flavour and fragrance of mushrooms are dependent on many volatile aroma compounds and their proportions. While the concentration of eight-carbon components and hydrocarbons was found the highest by using wood straw, amount of sulphur containing compounds and alcohols increased by agricultural wastes in this study. In addition, corn waste positively affected aldehyde compounds.

\section{Introduction}

Lentinula edodes is known as "shiitake mushroom" and is one of the most cultivated mushroom species in the world. World total mushroom production is 8993280 tons and the ranking is as follows: China with 6664606 tons, USA with 416050 tons, the Netherlands 300 000, Poland with 280232 tons and Spain 166250 (FAO, 2018). For shiitake mushroom, Royse (2014) reported that China is leader with over 4 million tons estimated and this corresponds to $90 \%$ of the total production. Li et al. (2019) emphasized that $70 \%$ of the shiitake mushroom production has been provided by China. In addition, China has been assessed as the most shiitake consuming country. Therefore, one may say that shiitake mushroom is one of the most produced and preferred mushroom species in the world. Although mushrooms have been known for both food and medicinal purposes since ancient times, with the increasing importance given to "functional foods", use of mushrooms for medical purposes has become even more important nowadays. Today, studies on determining of chemical composition of mushrooms are intensively conducted. These studies have been accelerated by using new technologies and also with becoming more accessible and more affordable of these technologies. The most advantage of shiitake mushroom is that it is both edible and medicinal. Some mushroom species are assessed as medicinal; however they may not be suitable for direct consumption, as for example Ganoderma lucidum, which has hard, woody texture and is therefore used in the form of tea or as dry extracts for addition to various beverages. To benefit from shiitake mushroom, it can be consumed directly or it can also be used as instant products such as tea and pills.

Different agricultural wastes are used in the production of mushrooms. These wastes vary according to their availability in different countries. Each country prefers to use their own wastes of agricultural products in the 
cultivation of mushrooms. Different agricultural wastes have been investigated in studies on shiitake mushroom cultivation, such as hazelnut husk-wheat straw-wheat branbeech sawdust (Özçelik and Pekşen, 2006), hazelnut huskwheat straw-beech wood chip-wheat bran mixtures (Özçelik and Pekșen, 2007), wheat straw-corn cobs-oak wood sawdust mixtures (Philippoussis et al., 2007), cocoa huskcotton waste-oak sawdust-wheat bran mixtures (Escobar et al., 2007), wheat straw-coir pith-poplar sawdust-teak sawdust-sal sawdust (Puri et al., 2011), eucalyptus sawdustrice bran-wheat bran-soybean bran (Casaril et al., 2011), oak sawdust-almond bark-poplar-walnut shell-linter-residue of textile fibers-olive waste-residue of guar-corn residueresidue of sunflower seed-residue of cotton-residue of grape mixture (Sözbir, 2014) and chickpea straw-corn stalk-alfalfa hay-sunflower head residue (Atila, 2019). In addition to all these, it was reported the use of wheat straw (Delpech and Olivier, 1991; Mata and Savoie, 1998; Savoie et al., 2000; Mata and Gaitán-Hernández, 2004; Philippoussis et al., 2007; Sharma et al., 2013; Gaitán-Hernández et al., 2014), paddy straw (Puri, 2012), coffee residues (Mata and GaitánHernández, 1994; Fan and Soccol, 2005), sunflower seeds hulls (Curvetto et al., 2004), vineyard pruning waste (Gaitán-Hernández et al., 2006), corn cobs (Philippoussis et al., 2007), sugar cane bagasse (Salmones et al., 1999), sawdust (Fan and Soccol, 2005; Royse and Sánchezi, 2007; Martínez-Guerrero et al., 2012) in a review study carried out on shiitake mushroom by Mata and Savoie (2018). Composition of these different agricultural wastes can affect taste and aroma of the mushrooms produced, as well as their quality and yield. Volatile aroma compounds are the major contributor to the characteristic mushroom flavour. The volatile compounds of shiitake mushroom have been studied by many researchers (Chen et al., 1984; Chen and Ho, 1986; Wu and Wang, 2000; Cho et al., 2003; Hiraide et al., 2004; Hiraide, 2006; Mata et al., 2014; Politowicz et al., 2018; Li et al., 2019). According to findings obtained from these studies, volatile components of shiitake mushroom are predominantly eight-carbon and sulphur compounds. There are many factors that affect the aroma content of mushrooms such as strains, substrate mixtures and mushroom maturity stage. Therefore, type and content of volatile compounds can vary significantly between two identical strains grown in different substrate mixtures. The aim of this study is to determine the effect of different agricultural substrate mixtures on the volatile aroma composition of shiitake mushroom.

\section{Material and Methods}

This study was carried out in Department of Horticulture (in Prof. Dr. Saadet BÜYÜKALACA Tissue Culture Laboratory and full climate-controlled mushroom growing room) of Cukurova University (Adana, Turkey). As spawn, strain " 4320 " obtained from Sylvan Cultivating Excellence was used.

\section{Substrate Preparation}

Poplar sawdust, wheat stalk, peanut shell, corn cob and vine pruning waste were selected as main ingredients. Wheat bran was added as additive materials $(5 \%)$. Oak sawdust used commonly in shiitake mushroom cultivation (Stamets, 1993) was assessed as control in the experiments.
The substrate materials were mixed in different ratios as indicated in Table 1.

Compost mixtures were filled into high temperature resistant polypropylene bags as $1 \mathrm{~kg}$ per bag. Sterilization of substrate materials was carried out in an autoclave at $121^{\circ} \mathrm{C}$ under $1.2 \mathrm{~atm}$ pressure for 90 minutes. Spawn inoculation was performed by mixing $50 \mathrm{~g}$ of spawn into per substrate bag in sterile bench. Substrate bags were placed in mushroom growing room set to $25 \pm 2{ }^{\circ} \mathrm{C}$ temperature and 70-80\% humidity for the first step aiming spawn development. After development of spawn, light was provided by using fluorescent lamps (300 lux) for 12 hours a day. Ventilation was performed 4-7 times per hour in the growing room to keep $\mathrm{CO}_{2}$ rate below $1000 \mathrm{ppm}$ and humidity was increased to $80-90 \%$. Figure 1 shows shiitake mushroom cultivated in A5 substrate mixture.

Table 1. Content of different substrate mixtures used in $L$. edodes production

\begin{tabular}{l|c}
\hline Substrate materials & SC \\
\hline Oak sawdust (control) & C \\
3 oak sawdust +1 wheat bran & A1 \\
3 poplar sawdust +1 wheat bran & A2 \\
3 wheat stalk +1 wheat bran & A3 \\
1 oak sawdust +1 poplar sawdust +1 wheat bran & A4 \\
1 oak sawdust +1 wheat stalk +1 wheat bran & A5 \\
3 peanut shell +1 wheat bran & A6 \\
3 corn cob +1 wheat bran & A7 \\
3 vine pruning waste +1 wheat bran & A8 \\
1 oak sawdust +1 peanut shell +1 wheat bran & A9 \\
1 oak sawdust +1 corn cob +1 wheat bran & A10 \\
1 oak sawdust +1 vine pruning waste +1 wheat bran & A11 \\
\hline
\end{tabular}

\section{SC: Substrate code}

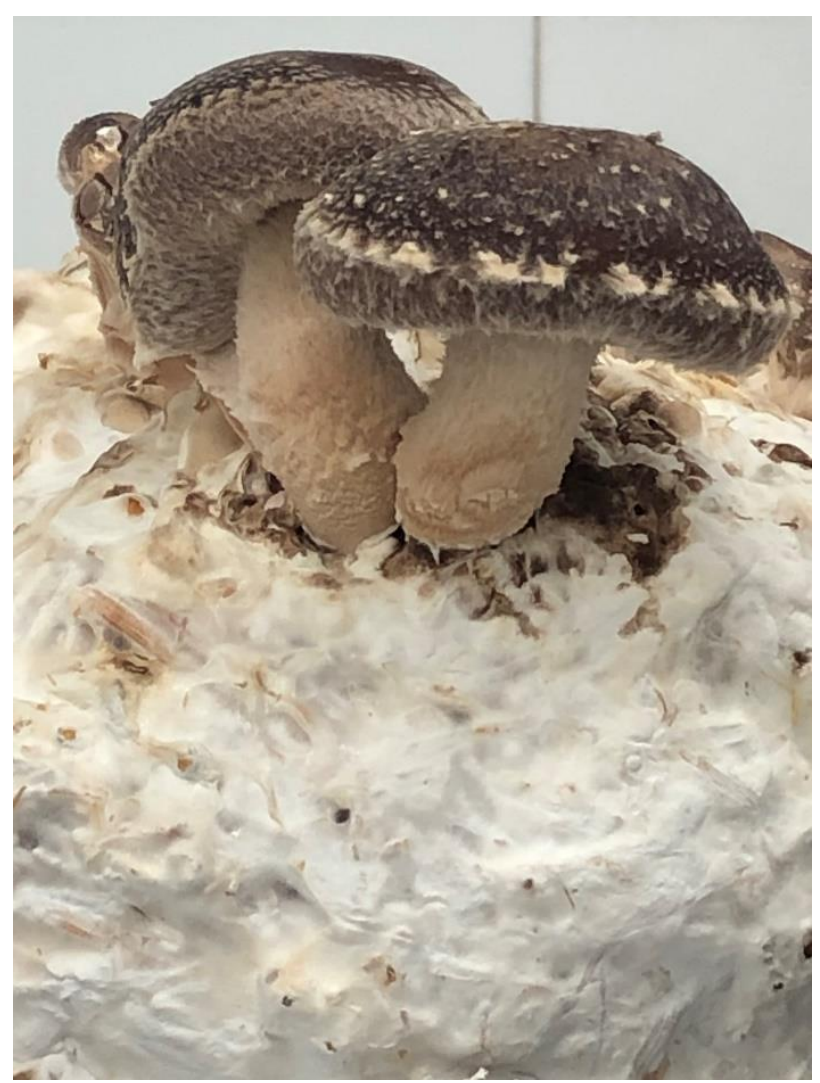

Figure 1. Lentinula edodes (shiitake) cultivated in A5 substrate mixture 


\section{Chemicals used for Volatile Aroma Analyses}

Retention indices were determined by using a mixture of $\mathrm{n}$-alkane standards ranging from $\mathrm{C} 8-\mathrm{C} 40$ and obtained from Sigma Aldrich (Stenheim, Germany). While acetaldehyde ( $\geq 99.5 \%$ purity), 3-methylbutanal $(\geq 99 \%$ purity), 3-octanone ( $\geq 98 \%$ purity), 3-octanol ( $\geq 98 \%$ purity), (E)-2-octenal ( $\geq 97 \%$ purity) and 1-octen-3-ol ( $\geq 98 \%$ purity) were provided from Merck (Darmstadt, Germany), dimethyl disulfide ( $\geq 98.5 \%$ purity), carbon disulphide ( $\geq 99 \%$ purity), 2-methyl-1-butanol ( $\geq 98 \%$ purity), 3-methyl-1-butanol ( $\geq 98.5 \%$ purity), 1-octen-3one ( $\geq 97$ purity), dimethyl trisulfide ( $\geq 98.5 \%$ purity), benzaldehyde ( $\geq 99 \%$ purity), 1-octanol ( $\geq 99.7 \%$ purity), benzyl alcohol ( $\geq 99.5 \%$ purity) and phenyl ethyl alcohol were obtained from Sigma Aldrich (Stenheim, Germany).

\section{Determination of Volatile Compounds}

HS-SPME technique combined with GC-MS was used to determine volatile compounds of the shiitake mushroom. Effects of different parameters such as extraction time $(15,30$ and 45 minutes) and extraction temperature $\left(30^{\circ} \mathrm{C}, 40^{\circ} \mathrm{C}, 50^{\circ} \mathrm{C}\right)$ were experimented for extraction efficiency. The highest peak area and the best extraction parameters (30 minutes of extraction time and $30^{\circ} \mathrm{C}$ extraction temperature) were applied based on detection number of peaks maintained consistency. Method of Palazzolo et al. (2017) was followed in preparation of samples with some small modifications. Fresh mushroom sample $(5 \mathrm{~g})$ was cut into slices, then placed into $20 \mathrm{~mL}$ vial with a PTFE/silicon septum (Supelco) and stored at $4{ }^{\circ} \mathrm{C}$ until the analysis. The volatile compounds were extracted by using $1 \mathrm{~cm} 50 / 30 \mu \mathrm{m}$ Divinylbenzene/Carboxen/Polydimethylsiloxane

(DVB/CAR/PDMS) StableFlex fibre (Supelco, Bellefonte, PA, USA). According to recommendation of the supplier, fibre was conditioned $\left(270^{\circ} \mathrm{C}\right.$ for $\left.60 \mathrm{~min}\right)$. For the extraction of volatile components, the fibre type $50 / 30 \mu \mathrm{m}$ DVB/CAR/PDMS suggested by many researchers (Tian et al., 2016; Politowicz et al., 2018; Li et al., 2019) was used because of its high sensitivity and extraction efficiency. Analyses of volatile compounds were carried out by using a 7890B Gas Chromatography (GC) equipped with 7000 triple quatro Mass Selective (MS) detector (Agilent Technologies). A DB-WAX Capillary column $(60 \mathrm{~m}$ lenght $\times 0.25 \mathrm{~mm}$ i.d. $\times 0.5 \mu \mathrm{m}$ thickness) was the stationary phase and flow rate of carrier gas (helium) was $1.5 \mathrm{~mL} / \mathrm{min}$. The oven temperature was firstly kept at $40^{\circ} \mathrm{C}$ for 4 minutes, then increased to $90^{\circ} \mathrm{C}$ at $3^{\circ} \mathrm{C} / \mathrm{min}, 130^{\circ} \mathrm{C}$ at $4^{\circ} \mathrm{C} / \mathrm{min}$ and $240^{\circ} \mathrm{C}$ at $5^{\circ} \mathrm{C} / \mathrm{min}$ and finally held at $240^{\circ} \mathrm{C}$ for 8 minutes. For the MSD, the same oven temperature program was applied. The MSD conditions were as follows: ionisation energy $70 \mathrm{Ev}$; mass range $\mathrm{m} / \mathrm{z} 30-300$ a.m.u; scan rate $2.0 \mathrm{scan} / \mathrm{s}$; interface temperature $250^{\circ} \mathrm{C}$ and source temperature $180^{\circ} \mathrm{C}$. The volatile compounds were identified by comparing the retention indices and their mass spectra from the DB-WAX column with those of a commercial spectra database (W9 N11.L, NIST98, flavour 2) and the internal library of instrument created from previous laboratory studies. The identification of some volatile compounds was confirmed by the injection of chemical standards into the GC-MS system under identical conditions. Retention indices for all volatile compounds detected were calculated using an n-alkane series. All analyses were performed in triplicates (Selli et al., 2006; Cho et al., 2007).

\section{Statistical Analysis}

The results of relative percentage (\%) of volatile compounds among different substrate mixtures containing different agricultural wastes were compared by the analysis of variance JMP version 5.0.1 (SAS Institute Inc., Cary, NC). A least significant difference test was done to examine the differences among groups. Comparisons that yielded $\mathrm{P} \leq 0.05$ were considered to be statistically significant.

\section{Result and Discussion}

HS-SPME technique combined with GC-MS allowed to identification of 41 volatile compounds isolated from shiitake mushroom cultivated in different substrate mixtures (Table 2a, b). These compounds were classified into six categories in terms of chemical functionality: 13 sulphur containing components (V1, V3, V10, V19, V29, V30, V31, V32, V33, V38, V39, V40 and V41), 7 eightcarbon components (V16, V17, V20, V21, V23, V25 and V26), 9 aldehydes (V2, V4, V5, V6, V11, V24, V27, V34 and V37), 4 alcohols (V14, V15, V35 and V36), 5 hydrocarbons (V8, V9, V12, V13 and V22), 2 ketones (V18 and V28) and 1 other compound (V7). Total quantity of volatile compounds and volatile compound profile showed differences in shiitake mushroom fruiting bodies grown in different substrate mixtures.

In studies carried out by Wu and Wang (2000) and Politowicz et al. (2018); 1-octen-3-ol, 3-octanone, dimethyl disulfide and dimethyl trisulfide, 1,2,4-trithiolane and 1,2,3,5,6-pentathiepane were identified as major volatile components in fresh shiitake mushroom. When we assessed our finding in terms of those compounds, the highest 1-octen-3-ol content was determined in the substrate mixture C (oak sawdust) with $48.66 \%$ and it was followed by A7 ( 3 corn cob +1 wheat bran) with $12.92 \%$, A2 (3 poplar sawdust +1 wheat bran) with $12.07 \%$, A1 (3 oak sawdust +1 wheat bran) with $9.25 \%$ and A5 (1 oak sawdust +1 wheat stalk +1 wheat bran) with $7.57 \%$. This compound was found to be $20.2 \%$ by the Politowicz et al. (2018) in the shiitake mushroom samples obtained from a company. The analyses of mushroom samples obtained from A5 substrate mixture (1 oak sawdust +1 wheat stalk +1 wheat bran) resulted with the highest amount of 3octanone with $12.05 \%$. The substrate mixtures A3 (3 wheat stalk +1 wheat bran), A4 (1 oak sawdust +1 poplar sawdust +1 wheat bran), A1 ( 3 oak sawdust +1 wheat bran), A2 (3 poplar sawdust +1 wheat bran) and C (oak sawdust) were considerably rich in terms of this volatile with $11.90 \%, 10.41 \%, 10.35 \%, 8.25 \%$ and $8.17 \%$, respectively.

The ranking for 3-octanol was as follows: A1 (3 oak sawdust +1 wheat bran) with $6.75 \%, \mathrm{~A} 3$ (3 wheat stalk + 1 wheat bran) with $6.37 \%$, A5 (1 oak sawdust +1 wheat stalk +1 wheat bran) with $6.20 \%$, A4 (1 oak sawdust +1 poplar sawdust +1 wheat bran) with $6.07 \%$ and A2 (3 poplar sawdust +1 wheat bran) with $4.73 \%$. Content of 3octanol changed between $0.97 \%$ and $6.75 \%$ among substrate mixtures. 
Table 2a. Volatiles and their amount detected in different substrate mixtures

\begin{tabular}{|c|c|c|c|c|c|c|c|c|c|}
\hline Code & Volatile Compounds & RT & RI & ID & $\mathrm{C}$ & A1 & A2 & A3 & A4 \\
\hline V1 & Methanethiol & 3,4 & 675 & RI,MS & nd & nd & nd & nd & nd \\
\hline $\mathrm{V} 2$ & Acetaldehyde & 3,5 & 716 & RI,MS,std & $0.48^{\mathrm{d}}$ & $0.65^{b}$ & $0.71^{\mathrm{a}}$ & $0.70^{\mathrm{a}}$ & $0.54^{\mathrm{c}}$ \\
\hline V3 & Carbon disulfide & 3,7 & 745 & RI,MS,std & $13.32^{\mathrm{a}}$ & $6.58^{\mathrm{bc}}$ & $5.84^{\mathrm{c}}$ & $6.70^{\mathrm{b}}$ & $1.60^{\mathrm{f}}$ \\
\hline V4 & Butanal & 4,5 & 904 & RI,MS & nd & 0.16 & 1.71 & 0 & 0.13 \\
\hline V5 & 2-Methylbutanal & 6,2 & 916 & RI,MS & nd & $0.52^{b}$ & $0.47^{\mathrm{b}}$ & $0.50^{\mathrm{b}}$ & $0.69^{a}$ \\
\hline V6 & 3-Methylbutanal & 6,3 & 930 & RI,MS,std & nd & $0.74^{\mathrm{de}}$ & $1.03^{\mathrm{c}}$ & $0.67^{\mathrm{ef}}$ & $1.32^{\mathrm{b}}$ \\
\hline V7 & Formamide & 6,9 & 940 & RI,MS & nd & $2.63^{\mathrm{b}}$ & $3.06^{\mathrm{a}}$ & $2.12^{\mathrm{d}}$ & $1.99^{\mathrm{d}}$ \\
\hline V8 & trans-1,3-octadiene & 7,4 & 954 & RI,MS & $0.35^{\mathrm{a}}$ & nd & $0.23^{\mathrm{b}}$ & nd & nd \\
\hline V9 & cis-1,3-octadiene & 7,4 & 958 & RI,MS & $0.94^{\mathrm{a}}$ & nd & $0.20^{\mathrm{b}}$ & nd & nd \\
\hline V10 & Dimethyl disulfide & 11,5 & 1047 & RI,MS,std & 2.401 & $8.07^{\mathrm{g}}$ & $10.43^{\mathrm{e}}$ & $8.14^{\mathrm{g}}$ & $10.49^{\mathrm{e}}$ \\
\hline V11 & Hexanal & 11,8 & 1077 & RI,MS & $0.88^{\mathrm{a}}$ & $0.30^{\mathrm{c}}$ & $0.32^{\mathrm{c}}$ & $0.26^{\mathrm{c}}$ & $0.25^{\mathrm{c}}$ \\
\hline V12 & Ethylbenzene & 13,6 & 1083 & RI,MS & nd & nd & nd & nd & $0.08^{\mathrm{b}}$ \\
\hline V13 & p-Xylene & 14,2 & 1128 & RI,MS & nd & $0.17^{\mathrm{d}}$ & $0,14^{\mathrm{e}}$ & $0.29^{b}$ & $0.30^{\mathrm{b}}$ \\
\hline V14 & 2-Methyl-1-butanol & 17,6 & 1132 & RI,MS,std & nd & nd & $0.55^{\mathrm{a}}$ & nd & $0.08^{b}$ \\
\hline V15 & 3-Methyl-1-butanol & 17,6 & 1207 & RI,MS,std & nd & $0.1^{\mathrm{e}}$ & $0.16^{\mathrm{c}}$ & $0.12^{\mathrm{d}}$ & $0.17^{\mathrm{c}}$ \\
\hline V16 & 3-Octanone & 19,6 & 1211 & RI,MS,std & $8.17^{\mathrm{c}}$ & $10.35^{\mathrm{b}}$ & $8.25^{\mathrm{c}}$ & $11.89^{\mathrm{a}}$ & $10.41^{\mathrm{b}}$ \\
\hline V17 & 1 -octen-3-one & 21,6 & 1259 & RI,MS,std & $0.85^{\mathrm{a}}$ & $0.51^{\mathrm{b}}$ & $0.28^{c}$ & $0.34^{\mathrm{c}}$ & $0.18^{\mathrm{d}}$ \\
\hline V18 & 4-Nonanone & 22,7 & 1263 & RI,MS & $0.43^{\text {fg }}$ & $2.48^{\mathrm{b}}$ & $2.01^{\mathrm{c}}$ & $3.43^{\mathrm{a}}$ & $3.52^{\mathrm{a}}$ \\
\hline V19 & Dimethyl trisulfide & 24,9 & 1279 & RI,MS,std & $1.42^{\mathrm{h}}$ & $10.23^{\mathrm{g}}$ & $17.38^{\mathrm{e}}$ & $10.30^{\mathrm{g}}$ & $14.14^{\mathrm{f}}$ \\
\hline V20 & 3-octanol & 25,6 & 1303 & RI,MS,std & $1.92^{\mathrm{d}}$ & $6.75^{\mathrm{a}}$ & $4.73^{c}$ & $6.37^{\mathrm{cab}}$ & $6.07^{\mathrm{b}}$ \\
\hline $\mathrm{V} 21$ & 2-octenal, (E)- & 26,8 & 1355 & RI,MS,std & $1.17^{\mathrm{a}}$ & $0.07^{b}$ & nd & nd & nd \\
\hline V22 & 1,3-dichlorobenzene & 27,3 & 1372 & RI,MS & $0.28^{\mathrm{a}}$ & $0.09^{c}$ & $0.08^{c}$ & $0.15^{\mathrm{b}}$ & $0.14^{\mathrm{b}}$ \\
\hline V23 & 1-octen-3-ol & 27,7 & 1400 & RI,MS,std & $48.67^{\mathrm{a}}$ & $9.25^{\mathrm{c}}$ & $12.07^{\mathrm{b}}$ & $3.48^{\mathrm{e}}$ & $4.26^{\mathrm{e}}$ \\
\hline V24 & Benzaldehyde & 30,1 & 1414 & RI,MS,std & $1.59^{\mathrm{h}}$ & $13.94^{\mathrm{ef}}$ & $11.10^{\mathrm{g}}$ & $16.12^{\mathrm{c}}$ & $14.62^{\mathrm{d}}$ \\
\hline $\mathrm{V} 25$ & 1-octanol & 31,4 & 1426 & RI,MS,std & $2.32^{\mathrm{a}}$ & $0.39^{\mathrm{bc}}$ & $0.39^{\mathrm{bc}}$ & $0.36^{\mathrm{bc}}$ & $0.22^{\mathrm{cd}}$ \\
\hline V26 & trans-2-octen-1-ol & 33,5 & 1493 & RI,MS & $1.69^{\mathrm{a}}$ & $0.21^{\mathrm{b}}$ & $0.15^{\mathrm{b}}$ & nd & $0.13^{\mathrm{b}}$ \\
\hline V27 & Benzeneacetaldehyde & 34,5 & 1528 & RI,MS & $0.88^{f}$ & $7.06^{\mathrm{c}}$ & $4.72^{\mathrm{d}}$ & $6.24^{\mathrm{c}}$ & $3.73^{\mathrm{e}}$ \\
\hline V28 & henone & 35,0 & 1584 & RI,MS & $0.69^{\mathrm{bc}}$ & $0.96^{\mathrm{a}}$ & $0.92^{\mathrm{a}}$ & $0.58^{\mathrm{c}}$ & $0.72^{\mathrm{b}}$ \\
\hline V29 & Methyl (methylthio)methyl disulfide & 35,5 & 1611 & RI,MS & $0.84^{\mathrm{g}}$ & $3.23^{\mathrm{ef}}$ & $2.56^{\mathrm{f}}$ & $2.87^{\mathrm{ef}}$ & $5.82^{\mathrm{d}}$ \\
\hline V30 & ethyl sulfide & 35,7 & 1622 & RI,MS & nd & nd & nd & nd & nd \\
\hline V31 & 1,3,5-tritl & 36,5 & 1634 & RI,MS & nd & nd & nd & nd & $0.23^{c}$ \\
\hline V32 & trasulfide & 38,6 & 1640 & RI,MS & nd & $0.14^{\mathrm{b}}$ & $0.13^{\mathrm{bc}}$ & nd & $0.11^{\mathrm{c}}$ \\
\hline V33 & 1,2,4-trithiolane & 38,8 & 1659 & RI,MS & $5.43^{c}$ & $6.06^{\mathrm{b}}$ & $4.09^{d}$ & $6.19^{\mathrm{b}}$ & $8.12^{\mathrm{a}}$ \\
\hline V34 & 2-phenylpropenal & 40,4 & 1714 & RI,MS & $0.27^{\mathrm{de}}$ & $2.0^{\mathrm{b}}$ & $0.53^{\mathrm{c}}$ & $2.63^{\mathrm{a}}$ & $0.20^{\text {def }}$ \\
\hline V35 & Benzyl alcohol & 42,7 & 1722 & RI,MS,std & $0.32^{f}$ & $2.53^{\mathrm{de}}$ & $2.26^{\mathrm{e}}$ & $2.98^{d}$ & $3.10^{\mathrm{d}}$ \\
\hline V36 & Phenylethyl alcohol & 43,6 & 1769 & RI,MS,std & nd & $0.27^{\mathrm{gh}}$ & $0.22^{\mathrm{h}}$ & $0.70^{\mathrm{e}}$ & $0.57^{\mathrm{f}}$ \\
\hline V37 & 2-phenyl-2-butenal & 44,1 & 1847 & RI,MS & nd & $0.36^{\mathrm{ef}}$ & $0.21^{\text {ef }}$ & $0.45^{\mathrm{de}}$ & $0.84^{\mathrm{d}}$ \\
\hline V38 & Methylthio(methylthio)methyl sulfone & 45,9 & 1881 & RI,MS & nd & $0.09^{\mathrm{cd}}$ & $0.12^{\mathrm{bc}}$ & $0.17^{\mathrm{bc}}$ & $0.21^{\mathrm{b}}$ \\
\hline V39 & 2,4,5-trithiahexane 2,2-dioxide & 45,9 & 1897 & RI,MS & $0.60^{\mathrm{e}}$ & $2.02^{\mathrm{d}}$ & $2.07^{\mathrm{d}}$ & $3.71^{\mathrm{c}}$ & $3.75^{\mathrm{c}}$ \\
\hline V40 & $1,2,4,5$-tetrathiane & 50,7 & 1970 & RI,MS & $1.26^{\mathrm{b}}$ & $0.87^{\mathrm{d}}$ & $0.63^{\mathrm{e}}$ & $0.94^{\mathrm{c}}$ & $0.66^{\mathrm{e}}$ \\
\hline V41 & Methane, tris(methylthio)- & 51,2 & 2218 & RI,MS & nd & $0,24^{\mathrm{e}}$ & $0.22^{\mathrm{e}}$ & $0.60^{\mathrm{d}}$ & $0.62^{\mathrm{d}}$ \\
\hline
\end{tabular}

The results of these three important compounds showed that the eight-carbon components are higher in the substrate mixtures including oak-poplar sawdust and wheat stalk except substrate A7 containing corn cob. When we considered that eight-carbon volatiles are usually high in wild mushroom species collected from forest (Taşkın, 2013; Taşkın et al., 2013; Bozok et al., 2015; Bozok et al., 2018; Taşkın et al., 2019), it seems quite normal that these compounds were found in higher amount in mushrooms grown on substrate mixtures containing wood sawdust than in those containing agricultural wastes.

A11 (oak sawdust +1 vine pruning waste +1 wheat bran), A10 (1 oak sawdust +1 corn cob +1 wheat bran), A9 (1 oak sawdust +1 peanut shell +1 wheat bran), A6 (3 peanut shell +1 wheat bran), A8 (3 vine pruning waste+ 1 wheat bran), A3 (3 wheat stalk +1 wheat bran), A2 (3 poplar sawdust +1 wheat bran), A5 (1 oak sawdust +1 wheat stalk +1 wheat bran) were rich in terms of dimethyl disulfide with $17.39 \%, 16.07 \%, 14.95 \%, 11.36 \%, 10.95 \%, 10.49 \%$, $10.43 \%$ and $9.34 \%$, respectively and amount of this volatile ranged between $2.39 \%$ and $17.39 \%$ in all substrate mixtures tested. For dimethyl trisulfide, A11 (oak sawdust +1 vine pruning waste +1 wheat bran) contained the highest amount with $38.96 \%$ and followed by A8 (3 vine pruning waste+ 1 wheat bran) with $34.24 \%$, A9 (1 oak sawdust +1 peanut shell +1 wheat bran) with $32.55 \%$, A10 (1 oak sawdust +1 corn cob +1 wheat bran) with $27.62 \%$ and A7 (3 corn cob +1 wheat bran) with $22.71 \%$. For all substrates, it ranged from $1.41 \%$ to $38.96 \%$. In contrast to the eight-carbon volatiles, sulphur containing compounds were higher in the substrate mixtures supplemented with agricultural wastes.

Maximum amount of 1,2,4-trithiolane was detected in A4 substrate (1 oak sawdust +1 poplar sawdust +1 wheat bran) with $8.12 \%$ and it was followed by A5 (1 oak sawdust +1 wheat stalk +1 wheat bran) with $7.75 \%$, A6 (3 peanut shell +1 wheat bran) with $7.59 \%$, A3 (3 wheat stalk +1 wheat bran) with $6.19 \%$ and A1 (3 oak sawdust +1 wheat bran) with $6.06 \%$. The amount of 1,2,4-trithiolane varied between $0.70 \%$ and $8.12 \%$. 
Table 2b. Volatiles and their amount detected in different substrate mixtures

\begin{tabular}{|c|c|c|c|c|c|c|c|c|c|}
\hline Code & Volatile Compounds & A5 & A6 & A7 & A8 & A9 & A10 & A11 & Sig. \\
\hline $\mathrm{V} 1$ & Methanethiol & nd & 0.97 & nd & nd & nd & nd & nd & $*$ \\
\hline $\mathrm{V} 2$ & Acetaldehyde & $0.72^{\mathrm{a}}$ & $0.11^{\mathrm{g}}$ & $0.26^{\mathrm{e}}$ & $0.21^{\mathrm{f}}$ & $0.19^{f}$ & $0.13^{\mathrm{g}}$ & nd & $*$ \\
\hline V3 & Carbon disulfide & $4.48^{\mathrm{d}}$ & $0.47^{\mathrm{c}}$ & $6.99^{\mathrm{b}}$ & $2.65^{\mathrm{e}}$ & $1.77^{\mathrm{f}}$ & $2.60^{\mathrm{e}}$ & $3.79^{\mathrm{d}}$ & $*$ \\
\hline V4 & Butanal & 0.13 & nd & nd & nd & nd & nd & nd & $*$ \\
\hline V5 & 2-Methylbutanal & $0.72^{\mathrm{a}}$ & nd & $0.31^{\mathrm{c}}$ & $0.41^{\mathrm{bc}}$ & nd & nd & nd & $*$ \\
\hline V6 & 3-Methylbutanal & $1.46^{\mathrm{a}}$ & nd & $0.56^{\mathrm{g}}$ & $0.70^{\mathrm{def}}$ & $0.60^{\mathrm{fg}}$ & $0.77^{\mathrm{d}}$ & $0.76^{\mathrm{de}}$ & $*$ \\
\hline V7 & Formamide & $2.34^{\mathrm{c}}$ & $1.53^{\mathrm{ef}}$ & $1.73^{\mathrm{e}}$ & $1.42^{\mathrm{fg}}$ & $1.13^{\mathrm{h}}$ & $1.22^{\mathrm{gh}}$ & $1.53^{\mathrm{ef}}$ & $*$ \\
\hline V8 & trans-1,3-octadiene & nd & nd & nd & nd & nd & nd & nd & $*$ \\
\hline V9 & cis-1,3-octadiene & nd & nd & nd & nd & nd & nd & nd & $*$ \\
\hline V10 & Dimethyl disulfide & $9.34^{\mathrm{f}}$ & $11.36^{\mathrm{d}}$ & $5.70^{\mathrm{h}}$ & $10.95^{\mathrm{de}}$ & $14.95^{\mathrm{c}}$ & $16.07^{\mathrm{b}}$ & $17.39^{\mathrm{a}}$ & $*$ \\
\hline V11 & Hexanal & $0.49^{b}$ & nd & nd & nd & nd & nd & nd & $*$ \\
\hline V12 & Ethylbenzene & $0.09^{\mathrm{a}}$ & nd & nd & nd & nd & nd & nd & $*$ \\
\hline V13 & p-Xylene & $0.33^{\mathrm{a}}$ & $0.15^{\mathrm{de}}$ & $0.15^{\mathrm{e}}$ & nd & nd & nd & $0.23^{\mathrm{c}}$ & $*$ \\
\hline V14 & 2-Methyl-1-butanol & $0.10^{\mathrm{b}}$ & nd & nd & nd & nd & nd & nd & $*$ \\
\hline V15 & 3-Methyl-1-butanol & $0.19^{\mathrm{b}}$ & nd & nd & nd & nd & $0.13^{\mathrm{d}}$ & $0.25^{\mathrm{a}}$ & $*$ \\
\hline V16 & 3-Octanone & $12.05^{\mathrm{a}}$ & $2.10^{\mathrm{h}}$ & $5.74^{\mathrm{d}}$ & $4.19^{\mathrm{e}}$ & $2.85^{\mathrm{fg}}$ & $2.35^{\mathrm{gh}}$ & $3.43^{\mathrm{f}}$ & $*$ \\
\hline V17 & 1-octen-3-one & $0.30^{c}$ & nd & nd & nd & nd & nd & nd & $*$ \\
\hline V18 & 4-Nonanone & $3.70^{\mathrm{a}}$ & $0.74^{\text {ef }}$ & $0.33^{\mathrm{g}}$ & $0.59^{\mathrm{efg}}$ & $0.63^{\text {efg }}$ & $0.83^{\mathrm{e}}$ & $1.20^{\mathrm{d}}$ & $*$ \\
\hline V19 & Dimethyl trisulfide & $14.02^{\mathrm{f}}$ & $14.59^{\mathrm{f}}$ & $22.71^{\mathrm{d}}$ & $34.24^{\mathrm{b}}$ & $32.55^{\mathrm{b}}$ & $27.62^{\mathrm{c}}$ & $38.96^{\mathrm{a}}$ & $*$ \\
\hline V20 & 3-octanol & $6.20^{\mathrm{b}}$ & $0.93^{\mathrm{g}}$ & $1.41^{\mathrm{ef}}$ & $1.83^{\mathrm{de}}$ & $0.97^{\mathrm{g}}$ & $1.08^{\mathrm{fg}}$ & $1.65^{\mathrm{de}}$ & $*$ \\
\hline V21 & 2-octenal, (E)- & $0.07^{\mathrm{b}}$ & nd & nd & nd & nd & nd & nd & $*$ \\
\hline $\mathrm{V} 22$ & 1,3-dichlorobenzene & $0.15^{b}$ & nd & nd & nd & nd & nd & nd & $*$ \\
\hline V23 & 1-octen-3-ol & $7.57^{\mathrm{d}}$ & $0.36^{\mathrm{g}}$ & $12.92^{\mathrm{b}}$ & $4.22^{\mathrm{e}}$ & $2.00^{\mathrm{f}}$ & $1.69^{\mathrm{f}}$ & $1.32^{\mathrm{fg}}$ & $*$ \\
\hline V24 & Benzaldehyde & $10.52^{\mathrm{g}}$ & $14.53^{\text {de }}$ & $21.76^{\mathrm{a}}$ & $17.79^{\mathrm{b}}$ & $16.79^{c}$ & $16.27^{\mathrm{c}}$ & $13.57^{\mathrm{f}}$ & $*$ \\
\hline $\mathrm{V} 25$ & 1-octanol & $0.40^{\mathrm{bc}}$ & $0.12^{\mathrm{cd}}$ & $0.60^{\mathrm{b}}$ & nd & nd & nd & nd & $*$ \\
\hline V26 & trans-2-octen-1-ol & $0.22^{\mathrm{b}}$ & nd & $0.33^{\mathrm{b}}$ & $0.18^{b}$ & nd & nd & nd & $*$ \\
\hline V27 & Benzeneacetaldehyde & $3.24^{\mathrm{e}}$ & $10.12^{\mathrm{a}}$ & $8.35^{\mathrm{b}}$ & $3.87^{\text {de }}$ & $1.12^{\mathrm{f}}$ & $0.94^{\mathrm{f}}$ & $0.21^{\mathrm{f}}$ & $*$ \\
\hline V28 & Acetophenone & $0.60^{\mathrm{bc}}$ & nd & $0.31^{\mathrm{d}}$ & nd & nd & nd & nd & $*$ \\
\hline V29 & Methyl (methylthio)methyl disulfide & $3.54^{\mathrm{e}}$ & $10.21^{\mathrm{a}}$ & $0.61^{\mathrm{g}}$ & $3.26^{\mathrm{ef}}$ & $6.97^{c}$ & $8.67^{\mathrm{b}}$ & $5.79^{d}$ & $*$ \\
\hline V30 & Benzyl methyl sulfide & nd & nd & $0.14^{\mathrm{b}}$ & nd & $0.12^{\mathrm{c}}$ & $0.19^{\mathrm{a}}$ & nd & $*$ \\
\hline V31 & 1,3,5-trithiane & nd & $0.43^{\mathrm{a}}$ & nd & nd & nd & $0.35^{\mathrm{b}}$ & nd & $*$ \\
\hline V32 & Dimethyl tetrasulfide & $0.08^{\mathrm{d}}$ & $2.24^{\mathrm{a}}$ & nd & nd & nd & nd & nd & $*$ \\
\hline V33 & 1,2,4-trithiolane & $7.75^{\mathrm{a}}$ & $7.59^{\mathrm{a}}$ & $0.70^{\mathrm{g}}$ & $1.22^{\mathrm{fg}}$ & $1.53^{\mathrm{f}}$ & $2.12^{\mathrm{e}}$ & $1.33^{\mathrm{f}}$ & $*$ \\
\hline V34 & 2-phenylpropenal & $0.35^{\mathrm{cd}}$ & $0.10^{\mathrm{ef}}$ & $0.39^{\mathrm{cd}}$ & nd & nd & nd & nd & $*$ \\
\hline V35 & Benzyl alcohol & $2.20^{\mathrm{e}}$ & $2.86^{\mathrm{d}}$ & $5.16^{\mathrm{b}}$ & $5.83^{\mathrm{a}}$ & $4.11^{\mathrm{c}}$ & $5.24^{\mathrm{b}}$ & $2.95^{\mathrm{d}}$ & $*$ \\
\hline V36 & Phenylethyl alcohol & $0.32^{\mathrm{g}}$ & $1.29^{c}$ & $0.62^{\text {ef }}$ & $1.18^{\mathrm{d}}$ & $1.74^{\mathrm{b}}$ & $2.17^{\mathrm{a}}$ & $1.14^{\mathrm{d}}$ & $*$ \\
\hline V37 & 2-phenyl-2-butenal & $0.54^{\mathrm{de}}$ & $0.36^{\mathrm{ef}}$ & $1.62^{\mathrm{c}}$ & $4.87^{\mathrm{a}}$ & $1.97^{\mathrm{bc}}$ & $2.17^{\mathrm{b}}$ & $0.42^{\mathrm{def}}$ & $*$ \\
\hline V38 & Methylthio(methylthio)methyl sulfone & $0.19^{\mathrm{b}}$ & $0.93^{\mathrm{a}}$ & nd & nd & nd & nd & nd & $*$ \\
\hline V39 & 2,4,5-trithiahexane 2,2-dioxide & $3.68^{c}$ & $12.31^{\mathrm{a}}$ & $0.75^{\mathrm{e}}$ & $0.39^{\mathrm{e}}$ & $6.35^{b}$ & $6.04^{\mathrm{b}}$ & $2.92^{\mathrm{cd}}$ & $*$ \\
\hline V40 & $1,2,4,5$-tetrathiane & $1.35^{\mathrm{a}}$ & $0.19^{f}$ & nd & nd & nd & $0.24^{\mathrm{f}}$ & nd & $*$ \\
\hline V41 & Methane, tris(methylthio)- & $0.56^{\mathrm{d}}$ & $3.41^{\mathrm{a}}$ & nd & nd & $1.67^{\mathrm{b}}$ & $1.08^{\mathrm{c}}$ & $1.15^{\mathrm{c}}$ & $*$ \\
\hline
\end{tabular}

In a study carried out by Mata et al. (2014), similar to our research, different substrate materials were tested in terms of aroma composition of shiitake mushroom. In the substrate mixtures containing barley straw and oak powder; 3-octanone, 1-octen-3-ol, benzeneacetaldehyde and benzaldehyde were found to be main volatiles with $79.72 \%, 18.93 \%, 0.76 \%$ and $0.57 \%$, respectively. The most important volatiles of sugar cane bagasse substrate were 3-octanone with 79.58\%, 1-octen-3-ol with $9.92 \%$, 2penten-1-ol with $9.32 \%$ and 1,2,4-trithiolane with $1.18 \%$. Mushroom samples obtained from substrate mixture prepared from beech tree litter and oak powder (Platanus mexicana) had 3-octanone (97.77\%) and 2-penten-1-ol (2.22\%). 3-octanone (71.40\%), 2-pentylfuran (11.78\%), limonene $(6.89 \%)$, benzeneacetaldehyde $(5.76 \%)$ and benzaldehyde $(4.15 \%)$ were the major volatiles in the oak wood sawdust. In our analyses, 1-octen-3-ol varied between $0.36 \%$ and $48.66 \%$ among substrate mixtures tested. It was detected between $9.92 \%$ and $18.93 \%$ in the research of Mata et al. (2014). Similarly, while amount of 3-octanone, benzaldehyde and benzeneacetaldehyde ranged from $2.10 \%$ to $12.05 \%$, from $2.02 \%$ to $21.76 \%$ and from $0.21 \%$ to $10.12 \%$ in this study, they changed between $71.40 \%$ and $97.77 \%, 0.57 \%$ and $4.14 \%$ and $0.76 \%$ and $5.76 \%$ respectively in the study carried out by Mata et al. (2014). The high amount of 3-octanone in their study may be related to a small number of volatiles (eight) detected.

Many different volatiles come together to create a different flavour and odour in mushrooms. Especially, the presence of 1-octen-3-ol known as mushroom alcohol and its amount is very important (Taşkın, 2013; Taşkın et al., 2013; Bozok et al., 2015; Bozok et al., 2018; Taşkın et al., 2019). Together with alcohols, sulphur containing compounds are known as effective in mushroom odour (Hiraide, 2006). 2,4-trithiolane and 1,2,4,6-tetrathiepane have been reported as major sulphur containing volatiles of shiitake mushroom in literature and also its use as an indicator in estimating odour of dried $L$. edodes has been 
emphasized (Hiraide, 2006). To increase sulphur content of shiitake mushroom, rice bran detected as rich in terms of sulphur was used in the cultivation of this mushroom species and then measured by Hiraide (2006). Among main volatiles detected in this study for fresh shiitake mushroom; 1-octen-3-ol, 3-octanone, 3-octanol, 1, 2, 4trithiolane, benzeneacetaldehyde and benzaldehyde are responsible for mushroom-butter-resinous, sweet fruity, cod liver oil, egg-garlic, fruity and bitter almond, respectively (Mata et al., 2014).

Maximum number of volatiles were obtained from A2 (3 poplar sawdust +1 wheat bran), A4 (1 oak sawdust +1 poplar sawdust +1 wheat bran) and A5 (1 oak sawdust +1 wheat stalk +1 wheat bran) substrate mixtures with 36 compounds and they were followed by A1 ( 3 oak sawdust +1 wheat bran) with 33 , A3 (3 wheat stalk +1 wheat bran) with 30 , A6 (3 peanut shell +1 wheat bran) with 26, A7 (3 corn cob +1 wheat bran) and C (oak sawdust) with $25, \mathrm{~A} 10$ (1 oak sawdust +1 corn cob +1 wheat bran) with $23, \mathrm{~A} 8$ (3 vine pruning waste+ 1 wheat bran), A9 (1 oak sawdust +1 peanut shell +1 wheat bran) and A11 (oak sawdust + 1 vine pruning waste +1 wheat bran) with 20 . Although there is significant difference between the substrate mixtures in terms of number of compounds, when content of the substrate mixtures is considered, there is no evidence to say that the substrate mixtures containing only agricultural wastes or only wood sawdust produce more compounds.

When we evaluated substrate mixtures in terms of eight-carbon components, the highest percentage was recorded in C (oak sawdust) with 64.79\%. Eight-carbon compounds are reported to be unstable during the drying process in literature ( $\mathrm{Li}$ et al., 2019). The rate for other substrates was as follows: A1 (3 oak sawdust +1 wheat bran) with $27.53 \%$, A5 (1 oak sawdust +1 wheat stalk +1 wheat bran) with $26.74 \%$, A2 (3 poplar sawdust +1 wheat bran) with $25.87 \%$, A3 (3 wheat stalk +1 wheat bran) with $22.44 \%$, A4 (1 oak sawdust +1 poplar sawdust +1 wheat bran) with $21.27 \%$, A7 (3 corn cob +1 wheat bran) with $21.05 \%$, A8 (3 vine pruning waste+ 1 wheat bran) with $10.42 \%$, A11 (oak sawdust +1 vine pruning waste +1 wheat bran) with $6.4 \%$, A9 (1 oak sawdust +1 peanut shell +1 wheat bran) with $5.82 \%$, A10 (1 oak sawdust +1 corn cob +1 wheat bran) with $5.11 \%$ and A6 (3 peanut shell + 1 wheat bran) with $3.51 \%$. As we emphasized before, it is clear that substrate mixtures containing wood straw are richer for eight-carbon components. The fact that having of the substrate mixture including only oak sawdust almost twice eight-carbon components than the substrate mixture nearest can be considered as an important result.

In contrast to the eight-carbon volatiles, agricultural wastes were richer than sulphur containing substrate mixtures such as A11 (oak sawdust + 1 vine pruning waste +1 wheat bran) with $71.33 \%$, A9 (1 oak sawdust +1 peanut shell +1 wheat bran) with $65.91 \%$, A10 (1 oak sawdust + 1 corn cob +1 wheat bran) with $64.98 \%$, A6 (3 peanut shell +1 wheat bran) with $64.70 \%$ and A8 (3 vine pruning waste +1 wheat bran) with $52.71 \%$. Ranking of the other substrates was as follows: A4 (1 oak sawdust +1 poplar sawdust +1 wheat bran) with $45.75 \%$, A5 (1 oak sawdust +1 wheat stalk +1 wheat bran) with $44.99 \%$, A2 (3 poplar sawdust +1 wheat bran) with $43.47 \%$, A3 (3 wheat stalk + 1 wheat bran) with $39.62 \%$, A7 (3 corn cob +1 wheat bran) with $37.60 \%$, A1 (3 oak sawdust +1 wheat bran) with $37.53 \%$ and C (oak sawdust) with $25.27 \%$.

The substrate mixtures including agricultural wastes seem again rich in terms of alcohols like this sulphur containing substrates: A10 (1 oak sawdust +1 corn cob + 1 wheat bran) with $7.54 \%$, A8 (3 vine pruning waste+ 1 wheat bran) with $7.01 \%$, A9 (1 oak sawdust +1 peanut shell +1 wheat bran) with $5.85 \%$, A7 (3 corn cob +1 wheat bran) with $5.78 \%$, A11 (oak sawdust +1 vine pruning waste +1 wheat bran) with $4.34 \%$, A6 (3 peanut shell +1 wheat bran) with $4.15 \%$, A4 (1 oak sawdust +1 poplar sawdust +1 wheat bran) with $3.92 \%$, A3 (3 wheat stalk + 1 wheat bran) with $3.80 \%$, A2 (3 poplar sawdust +1 wheat bran) with $3.19 \%$, A1 (3 oak sawdust +1 wheat bran) with $2.90 \%$, A5 (1 oak sawdust +1 wheat stalk +1 wheat bran) with $2.81 \%$ and $\mathrm{C}$ (oak sawdust) with $0.32 \%$.

Substrate mixture A7 (3 corn cob +1 wheat bran) was the most successful in containing aldehydes with $33.25 \%$ and followed by A8 (3 vine pruning waste +1 wheat bran) with $27.85 \%$, A3 (3 wheat stalk +1 wheat bran) with $27.57 \%$, A1 (3 oak sawdust +1 wheat bran) with $25.73 \%$, A6 (3 peanut shell + 1 wheat bran) with $25.46 \%$, A4 (1 oak sawdust +1 poplar sawdust +1 wheat bran) with $22.32 \%$, A2 (3 poplar sawdust +1 wheat bran) with $20.80 \%$, A9 (1 oak sawdust +1 peanut shell +1 wheat bran) with $20.67 \%$, A10 (1 oak sawdust +1 corn cob +1 wheat bran) with $20.28 \%$, A5 (1 oak sawdust +1 wheat stalk +1 wheat bran) with $18.17 \%$, A11 (oak sawdust +1 vine pruning waste + 1 wheat bran) with $14.96 \%$ and C (oak sawdust) with $4.1 \%$.

The substrate mixtures including wood sawdust were better in terms of hydrocarbons. Even no data was obtained from A8, A9 and A10 substrate mixtures supplemented agricultural wastes, the other mixtures could produce hydrocarbons [C (oak sawdust) with $1.57 \%$, A2 (3 poplar sawdust +1 wheat bran) with $0.65 \%$, A5 (1 oak sawdust + 1 wheat stalk +1 wheat bran) with $0.57 \%$, A4 (1 oak sawdust +1 poplar sawdust +1 wheat bran) with $0.52 \%$, A3 (3 wheat stalk +1 wheat bran) with $0.44 \%$, A1 (3 oak sawdust +1 wheat bran) with $0.26 \%$, A6 (3 peanut shell + 1 wheat bran) with $0.25 \%$, A11 (oak sawdust +1 vine pruning waste +1 wheat bran) with $0.23 \%$, A7 (3 corn cob +1 wheat bran) with $0.15 \%$ ].

For the two compounds described as ketone, A5 (1 oak sawdust +1 wheat stalk +1 wheat bran) with $4.30 \%$, A4 ( 1 oak sawdust +1 poplar sawdust +1 wheat bran) with $4.24 \%$, A3 (3 wheat stalk +1 wheat bran) with $4.01 \%$, A1 ( 3 oak sawdust +1 wheat bran) with $3.44 \%$ and A2 (3 poplar sawdust +1 wheat bran) with $2.93 \%$ were found to be better than A11 (oak sawdust +1 vine pruning waste + 1 wheat bran) with $1.20 \%$, C (oak sawdust) with $1.12 \%$, A10 (1 oak sawdust +1 corn cob +1 wheat bran) with $0.83 \%$, A6 (3 peanut shell +1 wheat bran) with $0.74 \%$, A9 (1 oak sawdust +1 peanut shell +1 wheat bran) with $0.63 \%$, A7 (3 corn cob +1 wheat bran) with $0.64 \%$ and A8 (3 vine pruning waste+ 1 wheat bran) with $0.59 \%$.

In a study carried out by $\mathrm{Li}$ et al. (2019) on effect of different agricultural wastes on the volatile profile of shiitake mushroom; sawdust, wheat bran, rice bran, soy bean pulp and corn meal were used and 82 volatiles were obtained including 7 sulphur containing components (49.55\%), 8 eight-carbon components (46.47\%), 19 aldehydes (35.11\%), 10 alcohols (22.82\%), 7 esters (46.26\%), 5 ketones (23.85\%), 10 acid components 
$(33.71 \%)$ and 16 other compounds $(58.18 \%)$. In their study, sulphur containing compounds and aldehydes were dominant except CS6 (sawdust, wheat bran and gypsum). While sulphur containing compounds were the highest in CS1 (sawdust, wheat bran and gypsum) and CS4 (sawdust, wheat bran, soy bean pulp and gypsum), maximum aldehydes were obtained from CS2 (sawdust, wheat bran, corn meal and gypsum). Agricultural wastes seem quite successful in obtaining sulphur containing compounds similar to our finding. However, while soy bean pulp, cereal bran, wheat bran and rice bran increased the amount of sulphur containing compounds, corn meal had positive effect on aldehyde concentration. Also, the highest data for aldehydes was recorded in A7 (3 corn cob +1 wheat bran). Both our A7 mixture and Li et al. (2019)' CS2 and CS7 mixtures contained corn wastes. This finding may mean that corn wastes have an important role on aldehydes content of shiitake mushroom.

In a very early study carried out by Chen and Ho in 1986, 18 noncyclic and cyclic sulphur containing volatiles (13 of them were assessed as new) were reported in fresh shiitake mushroom samples. Hiraide et al. (2004) emphasized that sulphur containing compounds have very important role in shiitake mushroom smell, especially in dried mushroom samples. 1,2,4-trithiolane and 1,2,4,6tetrathiepane were found as main volatiles, especially 1,2,4- trithiolane was suggested as indicator. Volatile profile of shiitake mushroom was determined at the young, immature, mature and old growing stages by Cho et al. (2003) and 129, 129, 111 and 120 volatiles were detected, respectively. 1-octen-3-ol, 3-octanol, 3-octanone and 4octen-3-one were identified as major components. They also realized that while 1-octen-3-ol content decreased with maturation, 3-octanone increased. The effect of $\mathrm{pH}$ on formation and concentration of volatiles was studied by Chen et al. (1984). It was found that while $\mathrm{pH}$ 5.0-5.5 was better for eight-carbon compounds, $\mathrm{pH} 7.0$ was suitable for sulphurous compounds in shiitake mushroom. The researchers considered that two different enzyme systems were active for these different volatile groups.

\section{Conclusion}

In this study, the changes on the aroma profile with the use of different agricultural materials in shiitake mushroom cultivation were assessed. Oak sawdust, poplar sawdust, wheat bran, wheat stalk, peanut shell, corn cob and vine pruning waste were used as combination with each other at different ratios. Important results were obtained when experiments were completed: (i) eight-carbon components and hydrocarbons were detected at higher amount in the substrate mixtures including wood straw than agricultural wastes (ii) sulphur containing substrates and alcohols were found rather than in the substrate mixtures including agricultural wastes (iii) corn wastes seem to have considerable effect on aldehydes content (iii) dimethyl trisulfide, benzaldehyde, dimethyl disulfide, 1-octen-3-ol and 3-octanone were identified as major volatile components. Overall, the substrate material has significantly affected the volatile aroma composition of the mushroom. Mushrooms are consumed because of their unique taste and aroma. Therefore, the results obtained from this study are important in terms of practice. For example, sulphur containing component are very important in the formation of odour in the shiitake mushroom and therefore, it is very valuable to have knowledge which substrate materials can lead to increase in the amount of the sulphur containing volatiles. The availability and efficiency of agricultural wastes in mushroom cultivation will reduce the need for wood sawdust. There are two important reasons for wanting to reduce the use of wood sawdust in mushroom production. The first is the difficulty of the availability of wood sawdust. The second is that while wood may be used in different production areas, agricultural wastes do not have different uses.

\section{Acknowledgment}

The authors would like to thank to Scientific and Technological Research Council of Turkey (TÜBİTAK) (Project code: 1190158) for supporting financially this study.

\section{References}

Atila F. 2019. Compositional changes in lignocellulosic content of some agro-wastes during the production cycle of shiitake mushroom. Scientia Horticulturae, 245: 263-268.

Bozok F, Zarifikhosroshahi M, Kafkas E, Taşkın H, Buyukalaca S. 2015. Comparison of Volatile Compounds of Fresh Boletus edulis and B. pinophilus in Marmara Region of Turkey. Not. Bot. Horti Agrobo., 43(1): 192-195.

Bozok F, Kafkas E, Büyükalaca S. 2018. Determination of Volatile Aroma Composition of Suillus collinitus (Fr.) Collected from Adana Province of Turkey. Turkish Journal of Agriculture - Food Science and Technology, 6(4): 486-489.

Casaril KBPB, Kasuya MCM, Vanetti MCD. 2011. Antimicrobial activity and mineral composition of shiitake mushrooms cultivated on agricultural waste. Brazilian Archives of Biology and Technology, 54(5): 991-1002.

Chen CC, Chen SD, Chen JJ, Wu CM. 1984. Effects of pH value on the formation of volatiles of shiitake (Lentinus edodes), an edible mushroom. J. Agric. Food Chem., 32: 999-1001.

Chen CC, Ho CT. 1986. Identification of Sulfurous Compounds of Shiitake Mushroom (Lentinus edodes Sing.). J. Agric. Food Chem., 34: 830-833.

Cho DB, Seo HY, Kim KS. 2003. Analysis of the Volatile Flavor Compounds Produced during the Growth stages of the Shiitake mushrooms (Lentinus edodes). J. Food Sci. Nutr., 8: 306-314.

Cho IH, Lee SM, Kim SY, Choi HK, Kim KO, Kim YS. 2007. Differentiation of aroma characteristics of pine-mushrooms (Tricholoma matsutake Sing.) of different grades using gas chromatography-olfactometry and sensory analysis. J. Agr. Food. Chem. 55(6): 2323-2328.

Curvetto NR, Gonzalez Matute R, Figlas D, Delmastro S. 2004. Sunflower seed hulls. Part II. Oyster Mushrooms. Oyster Mushroom Cultivation. Mushroom Growers' Handbook 1. MushWorld, Seul, pp. 95-100.

Delpech P, Olivier JM. 1991. Cultivation of shiitake on straw based pasteurized substrates. Mushroom Science, 13: 523528.

Escobar VV, Pérez AM, Arredondo C. 2007. Evaluación de la producción del hongo Lentinula edodes Pegler en bloques sintéticos a base de residuos agroindustriales. Ingeniería y Ciencia, 3(6): 23-39.

Fan L, Soccol CR. 2005. Shiitake Bag Cultivation: Coffee Residues. Mushroom Growers' Handbook 2: Shiitake Cultivation. Seul, Korea: MushWorld, pp. 73-151. ISSN 1739-1377. 
FAO, 2018. Food and Agriculture Organization of the United Nations. FAOSTAT. http://www.fao.org/faostat/en/ \#data/QC Last access date: 28/02/2020.

Gaitán-Hernández R, Cortés N, Mata G. 2014. Improvement of yield of the edible and medicinal mushroom Lentinula edodes on wheat straw by use of supplemented spawn. Braz. J. Microbiol., 45(2): 467-474.

Gaitán-Hernández R, Esqueda M, Gutiérrez A, Sánchez A, Beltrán-García M, Mata G. 2006. Bioconversion of agrowastes by Lentinula edodes: the high potential of viticulture residues. Appl. Microbiol. Biotechnol., 71: 432439.

Hiraide, M, Miyazaki Y, Shibata Y. 2004. The smell and odorous components of dried shiitake mushroom, Lentinula edodes I: relationship between sensory evaluations and amounts of odorous components. J. Wood. Sci., 50: 358-364.

Hiraide M. 2006. The smell and odorous components of dried shiitake mushroom, Lentinula edodes III: substances that increase the odorous compound content. J. Wood Sci. doi: 10.1007/s10086-005-0758-Z

Li W, Wang J, Chen W, Yang Y, Zhang J, Feng J, Yu H, Li Q. 2019. Analysis of volatile compounds of Lentinula edodes grown in different culture substrate formulations. Food Research International, 125. https://doi.org/10.1016/ j.foodres.2019.108517

Martínez-Guerrero MA, Sihuanca D, Macías-López A, PérezLópez RI, Martínez-Madrigal JD, López-Olguín JF. 2012. Characterization and production of shiitake (Lentinula edodes) in Mexico using supplemented sawdust. African Journal of Biotechnology, 11(46): 10582-10588.

Mata G, Gaitán-Hernández R. 1994. Avances en el cultivo del shiitake en pulpa de café. Rev. Iberoam. Micol., 11: 90-91.

Mata G, Savoie JM. 1998. Extracellular enzyme activities in six Lentinula edodes strains during cultivation in wheat straw. World Journal of Microbiolgy \& Biotechnology, 14: 513519.

Mata G, Gaitán-Hernández R. 2004. Cultivation of the Edible Mushroom Lentinula edodes (Shiitake) in Pasteurized Wheat Straw: Alternative Use of Geothermal Energy in Mexico. Engineering in Life Science, 4(4): 363-367.

Mata G, Valdez K, Mendoza R, Trigos Á. 2014. HS/GC-MS Analyzed Chemical Composition of the Aroma of Fruiting Bodies of Two Species of Genus Lentinus (Higher Basidiomycetes). International Journal of Medicinal Mushrooms, 16(5): 477-484.

Mata G, Savoie JM. 2018. Shiitake Cultivation on Straw: An Alternative for Subtropical Regions. In: Sánchez JE, Mata G, Daniel J. Royse DJ. (editors). Updates on Tropical Mushrooms. Basic and Applied Research. 1st. edition. San Cristóbal de Las Casas, Chiapas, México: El Colegio de la Frontera Sur, pp. 115-139.

Özçelik E, Peşken A. 2006. Lentinus edodes Yetiştiriciliğinde Findık Zurufundan Hazırlanan Farklı Yetiștirme Ortamlarının Verim ve Bazı Mantar Özelliklerine Etkileri. OMÜ Zir. Fak. Dergisi, 21(1): 65-70.

Özçelik E, Peşken A. 2007. Hazelnut husk as a substrate for the cultivation of shiitake mushroom (Lentinula edodes). Bioresource Technology, 98(14): 2652-2658.

Philippoussis A, Diamantopoulou Israilides P, Israilides C. 2007. Productivity of agricultural residues used for the cultivation of the medicinal fungus Lentinula edodes. International Biodeterioration \& Biodegradation, 59(3): 216-219.

Palazzolo E, Saiano F, Laudicina VA, Gargano ML, Venturella G. 2017. Volatile organic compounds in wild fungi from Mediterranean forest ecosystems. Jouenal of Essential Oil Research, 29(5): 385-390.
Politowicz J, Lech K, Lipan L, Figiel A, Carbonell-Barrachina ÁA, 2018. Volatile composition and sensory profile of shiitake mushrooms as affected by drying method. Journal of the Science of Food and Agriculture, 98(4): 1511-1521.

Puri S, Bhatt R, Mishra KK. 2011. Cultivation of Lentinula edodes (Berk.) Pegler on Sawdust Substrates and Agricultural Wastes. International Journal of Science and Nature, 2(4): $752-756$.

Puri SK. 2012. Vegetative growth and fruiting induction of Lentinula edodes strains on different substrates. The Bioscan, 7: 9-12.

Royse DJ, Sánchez JE. 2007. Ground wheat straw as a substitute for portions of oak wood chips used in shiitake (Lentinula edodes) substrate formulae. Bioresour. Technol., 98(11): 2137-2141.

Royse DJ. 2014. A global perspective on the high five: Agaricus, Pleurotus, Lentinula, Auricularia \& Flammulina. In: Singh $\mathrm{M}$ (editor). Proceedings of the 8th International Conference on Mushroom Biology and Mushroom Products (ICMBMP8). New Delhi, India, 19-22 November 2014, pp. $1-6$.

Salmones D, Mata G, Ramos LM, Waliszewski KN. 1999. Cultivation of shiitake mushroom, Lentinula edodes, in several lignocellulosic materials originating from the subtropics. Agronomie, 19: 13-19.

Savoie JM, Delpech P, Billette C, Mata G. 2000. Inoculum adaptation changes the outcome of the competition between Lentinula edodes and Trichoderma spp. during shiitake cultivation on pasteurized wheat straw. In: Van Griensven LJLD (editor). Science and cultivation of edible fungi. Proceedings of the 15th International Congress on the Science and Cultivation of Edible Fungi, Maastricht, Netherlands, 1519 May 2000, pp.667-674.

Selli S, Canbas A, Cabaroglu T, Erten H, Günata Z. 2006. Aroma components of cv. Muscat of Bornova wines and influence of skin contact treatment. Food Chemistry, 94(3): 319-326.

Sharma S, Khanna PK, Kapoor S. 2013. Effect of supplementation of wheat bran on the production of shiitake (Lentinus edodes (Berk) Peglar) using wheat straw and saw dust substrates. The Bioscan, 8(3): 817-820.

Sözbir GD. 2014. Farklı Besin Ortamlarının Lentinus edodes (Shiitake) Mantarında Verim, Lentinan ve Kimyasal Bileşimine Etkileri. Doktora Tezi, Kahramanmaraş Sütçü İmam Üniversitesi Fen Bilimleri Enstitüsü Orman Endüstri Mühendisliği Anabilim Dalı, $175 \mathrm{~s}$.

Stamets P. 1993. Growing Gourmet and Medicinal Mushrooms. Berkley: Ten Speed Press. ISBN 0-89815-608-4.

Taşkın H. 2013. Detection of Volatile Aroma Compounds of Morchella by Headspace Gas Chromatography Mass Spectrometry (HS-GC/MS). Not. Bot. Horti Agrobo., 41(1): 122-125.

Taşkın H, Baktemur G, Kafkas E, Büyükalaca S. 2013. Gyromitra Mantarının Uçucu Aroma Bileşenlerinin Tepe Boşluğu Gaz Kromotografisi Kütle Spektroskopisi (HS-GC/MS) Tekniği ile Belirlenmesi. Turkish Journal of Agriculture - Food Science and Technology, 1(1): 8-11.

Taşkın H, Çelik ZD, Bozok F, Cabaroğlu T, Büyükalaca S. 2019. First Report on Volatile Composition of Tricholoma anatolicum in Comparison with Tricholoma caligatum. Records of Natural Products, 6: 446-455.

Tian Y, Zhao Y, Huang J, Zeng H, Zheng B. 2016. Effects of Different Drying Methods on the Product Quality and Volatile Compounds of Whole Shiitake Mushrooms. Food Chemistry, 197: 714-722.

Wu CM, Wang Z. 2000. Volatile Compounds in Fresh and Processed Shiitake Mushrooms (Lentinus edodes Sing.). Food Science and Technology Research, 6(3): 166-170. 